\title{
Regional Anesthesia Practice in Neurosurgery
}

\author{
Parmod K. Bithal ${ }^{1,0} \quad$ Girija P. Rath ${ }^{1}$ \\ ${ }^{1}$ Department of Neuroanaesthesiology and Critical Care, All India \\ Institute of Medical Sciences, New Delhi, India \\ J Neuroanaesthesiol Crit Care 2021;8:85-86.
}

Maintenance of cerebral and spinal cord perfusion is the cornerstone for the safety of any neurosurgical procedure, and it depends upon stable hemodynamics. ${ }^{1}$ Fluctuation in blood pressure (BP) in patients with impaired cerebral autoregulation may impact cerebral blood flow (CBF), and thus, perioperative morbidity and mortality. ${ }^{2}$ Inadequate analgesia under general anesthesia (GA) is one major factor that causes episodic surges in BP. Anesthesiologists rely primarily on opioids for intraoperative analgesia during neurosurgery. Quick postoperative recovery after craniotomy is critically important and highly desirable. However, the opioids may delay recovery from anesthesia, thereby preventing immediate postoperative neurological assessment. Opioids may also cause respiratory depression with its attendant sequel, as well as postoperative nausea and vomiting (PONV).

Local anesthetic infiltration of the scalp for craniotomies, in combination with GA, was first described by Harvey Cushing and George Crile in the early 1900s. ${ }^{1}$ Modern neuroanesthesia with extensive neuromonitoring capabilities and safer anesthetic agents restricted the utility of regional anesthesia (RA) in neurosurgical patients. However, the resurgence of interest in awake craniotomy with expanding neurosurgical indications and awareness of the side effects of opioids has demonstrated that there is still a place for RA in neurosurgery, not only for craniotomy procedures but also for many spinal procedures. RA alone is not only valuable to facilitate safer surgery in some specific groups of neurosurgical procedures, but it is also a key modality of providing postoperative analgesia, thereby neuroanesthesiologists can afford to shun their reliance on opioids. More importantly, RA application has minimal effects on hemodynamics.

Scalp nerve block (SNB) is the principal modality of RA used for awake craniotomy and to provide postoperative analgesia. SNB is easy to achieve and requires knowledge of anatomical landmarks for innervation of the forehead and scalp by various sensory branches of the trigeminal nerve. For successful SNB, bilateral branches of trigeminal nerves should be blocked. However, the posterior scalp is innervated by cranial spinal nerves. SNB provides effective and prolonged postoperative analgesia in adults as well as in small children. ${ }^{3,4}$ SNB also facilitates "transitional analgesia" following remifentanil-based intraoperative analgesia. ${ }^{5}$ SNB largely obviates hemodynamic perturbations from Mayfield clamp application, which are not effectively attenuated by opioid administration under GA. ${ }^{6}$ Scalp block is associated with improved progression-free survival of patients receiving primary glioma resection. ${ }^{7}$

By reducing the intraoperative opioid requirement, SNB facilitates immediate neurological assessment postoperatively. Ropivacaine having selective action on the sensory A $\mathrm{\delta}$ and $\mathrm{C}$ fibers (because of rich innervation of the scalp by these nerve fibers) is a favored agent for SNB, in the strength of $0.75 \%$. The effect of adjuvants such as opioids, dexmedetomidine, dexamethasone, and magnesium sulfate has been investigated to improve the quality and duration of the block, showing varying results.

Spine surgery, which constitutes a significant proportion of the neurosurgical population, may be simple (single or two levels of discectomy) or complex (requiring instrumentation). Neuraxial anesthesia is an exciting proposition for single or two levels of discectomy. A review of the literature suggested that both RA and GA are safe and effective techniques for lumbar spine surgery and that RA may prove a better alternative to GA particularly, for patients who are at higher risk of complications of GA. ${ }^{8}$ Epidural anesthesia is also a substitute for spinal anesthesia in simple lumbar spine surgery. ${ }^{9}$ Neuraxial anesthesia is associated with cost reduction to the hospital as well as to the patients, diminished blood loss, lower incidence of hemodynamic fluctuations, reduced need for postoperative analgesia, shorter hospital stay, lower incidence of urinary retention, the fewer incidence of PONV, and lessened incidence of thromboembolism. In addition, patients are mildly sedated during surgery, which allows addressing position-related discomfort. Due to the limited duration of action of spinal anesthesia, this technique must be eschewed in procedures expected to last longer than 3 hours and also when a less experienced neurosurgeon conducts the surgery. The possibility of losing the airway in a prone position with limited choices in useful airway devices and an inability to perform normal airway rescue maneuvers must be taken into account when considering this technique. ${ }^{10}$ Additionally, there is the possibility of intrathecal migration of local anesthetic if dura mater is torn during surgery. The pain of complex lumbar spine surgery can be effectively prevented by erector spinae plane block (ESPB). However, the current evidence is insufficient to support the routine use of ESPB for lumbar spine surgery. High-quality randomized controlled trials are urgently needed. ${ }^{11}$

\author{
Address for correspondence Girija \\ Prasad Rath, MD, DM, Department \\ of Neuroanaesthesiology \\ and Critical Care, All India \\ Institute of Medical Sciences, \\ New Delhi 110029, India \\ (e-mail: girijarath.aiims@gmail. \\ com).
}

DOI https://doi.org/ 10.1055/s-0041-1734402 ISSN 2348-0548 
The neuraxial block is not restricted only to adult lumbar spine surgery but has been found useful for repairing meningomyelocele. ${ }^{12}$ Neonates born with this neural tube defect usually have hydrocephalus that may make airway management difficult. ${ }^{13}$ Spinal anesthesia ensures patent airway, avoids any direct pressure to the exposed neural placode, decreases postoperative apneic episodes, and attenuates the surgical stress response. Viscomi et al reported successful repair of meningomyelocele in 14 patients. ${ }^{12}$

Carotid artery stenosis is now being diagnosed frequently due to increasing life expectancy. The American Heart Association guidelines recommend carotid endarterectomy (CEA) in symptomatic patients with carotid artery stenosis of 50 to $99 \%$ if the risk of perioperative stroke or death is less than $6 \%{ }^{14}$ Elderly patients (more than 75 years of age) derive more benefits from this procedure than the younger ones. CEA can be performed either under GA or RA with cervical plexus block (CPB); the $\mathrm{CPB}$ is the preferred technique.

The cervical plexus arises from the ventral rami of $\mathrm{C} 1$ to C4 nerves, divided into superficial plexus and deep plexus. The deep branches are motor, while the superficial branches are sensory and supply the skin and subcutaneous tissues of the neck and posterior aspect of the head. Location of peripheral nerves with nerve stimulators and ultrasound has refined the technique of $\mathrm{CPB} .{ }^{15}$ With $\mathrm{CPB}$, an awake patient can self-monitor the satisfactory $\mathrm{CBF}$ throughout cross-clamping of the carotid artery; it provides predictable hemodynamic control and reduces hospital stay. CPB for CEA is particularly useful in healthcare facilities with limited neuromonitoring resources. CPB has also been used in anterior cervical discectomy and fixation (ACDF). However, the GA is superior to CPB in achieving better intraoperative hemodynamic stability and higher patient satisfaction with adequate intraoperative analgesia for patients undergoing ACDF. However, GA entails longer surgical and anesthesia time, may require more postoperative analgesia, and increased cost of anesthesia. ${ }^{16}$

Possible concerns with $\mathrm{CPB}$ in this procedure include remote access to the upper airway, conversion to GA, if needed, is difficult, and the need for patient cooperation. Ultrasound-guided deep CPB may allow a reduction in complications from inadvertent subarachnoid deposition of local anesthetic or injection of the agents into the vertebral artery, and respiratory distress due to the entrapment of the local anesthetic agent in paravertebral space and the direct spread of the anesthetic injection around the cervical nerve plexus. ${ }^{17}$

Apart from an alternative option to GA in a certain neurosurgical scenario, RA has been employed regularly in neurosurgical practice, in line with the upcoming concept of pre-emptive analgesia and its role in perioperative hemodynamic stability and early postoperative recovery. It should also be borne in mind that acute and chronic postneurosurgical pain has been extensively studied, and RA has a major role in their management. Nonetheless, the nerve blocks are an excellent analgesic option for polytrauma patients managed in the neurointensive care units. Pain is an important factor affecting intracranial pressure (ICP) in such patients, and appropriate analgesia helps control the ICP.

This narrative is a further testament to the fact that RA is an underutilized asset in the armamentarium of neuroanesthesiologists. if employed, in cooperative patients or patients with multiple systemic comorbidities, RA would lead to better patient outcomes with preservation of hospital resources. The use of RA in neurosurgery may be under-reported. We want to emphasize the utility and encourage its widespread use and documentation.

\section{Conflict of Interest}

None declared.

\section{References}

1 OsbornI,SebeoJ. “Scalp block" during craniotomy: a classictechnique revisited. J Neurosurg Anesthesiol 2010;22(3):187-194

2 Flexman AM, Ng JL, Gelb AW. Acute and chronic pain following craniotomy. Curr Opin Anaesthesiol 2010;23(5):551-557

3 Akhigbe T, Zolnourian A. Use of regional scalp block for pain management after craniotomy: review of literature and critical appraisal of evidence. J Clin Neurosci 2017;45:44-47

4 Festa R, Tosi F, Pusateri A, et al. The scalp block for postoperative pain control in craniosynostosis surgery: a case control study. Childs Nerv Syst 2020;36(12):3063-3070

5 Ayoub C, Girard F, Boudreault D, Chouinard P, Ruel M, Moumdjian R. A comparison between scalp nerve block and morphine for transitional analgesia after remifentanil-based anesthesia in neurosurgery. Anesth Analg 2006;103(5):1237-1240

6 Levin R, Hesselvik JF, Kourtopoulos H, Vavruch L. Local anesthesia prevents hypertension following application of the Mayfield skull-pin head holder. Acta Anaesthesiol Scand 1989;33(4):277-279

7 Sung CH, Tsuang FY, Shih CC, et al. Scalp block was associated with improved recurrence profiles in patients undergoing primary glioma resection surgery. J Neurosurg Anesthesiol 2021;33(3):239-246

8 De Rojas JO, Syre P, Welch WC. Regional anesthesia versus general anesthesia for surgery on the lumbar spine: a review of the modern literature. Clin Neurol Neurosurg 2014;119:39-43

9 Albayrak S, Erol FS, Demirel I, Ayden O, Ucler N. Lumbar disc surgery with epidural anesthesia: a review of 700 cases. Turk Neurosurg 2016;26(3):399-403

10 Meng T, Zhong Z, Meng L. Impact of spinal anaesthesia vs. general anaesthesia on peri-operative outcome in lumbar spine surgery: a systematic review and meta-analysis of randomised, controlled trials. Anaesthesia 2017;72(3):391-401

11 Qiu Y, Zhang TJ, Hua Z. Erector spine plane block for lumbar spinal surgery: a systematic review.J Pain Res 2020;13:1611-1619

12 Viscomi CM, Abajian JC, Wald SL, Rathmell JP, Wilson JT. Spinal anesthesia for repair of meningomyelocele in neonates. Anesth Analg 1995;81(3):492-495

13 Miller V, Rockoff MA, Neurosurgical anesthesia. In. Cote CJ, ed. A Practice of Anesthesia in Infants and Children. Philadelphia: WB Saunders; 1993:353-354

14 Kernan WN, Ovbiagele B, Black HR, et al. American Heart Association Stroke Council, Council on Cardiovascular and Stroke Nursing, Council on Clinical Cardiology, and Council on Peripheral Vascular Disease. Guidelines for the prevention of stroke in patients with stroke and transient ischemic attack: a guideline for healthcare professionals from the American Heart Association/American Stroke Association. Stroke 2014;45(7):2160-2236

15 Stoneham MD, Stamou D, Mason J. Regional anaesthesia for carotid endarterectomy. Br J Anaesth 2015;114(3):372-383

16 Wang H, Ma L, Yang D, et al. Cervical plexus anesthesia versus general anesthesia for anterior cervical discectomy and fusion surgery: a randomized clinical trial. Medicine (Baltimore) 2017;96(7):e6119

17 Saranteas T, Kostopanagiotou GG, Anagnostopoulou S, Mourouzis K, Sidiropoulou T. A simple method for blocking the deep cervical nerve plexus using an ultrasound-guided technique. Anaesth Intensive Care 2011;39(5):971-972 